Mathematical Modelling and Analysis

Volume 18 Number 2, April 2013, 204-218

http://dx.doi.org/10.3846/13926292.2013.781068

(c) Vilnius Gediminas Technical University, 2013
Publisher: Taylor\&Francis and VGTU

http://www.tandfonline.com/TMMA

Print ISSN: 1392-6292

Online ISSN: 1648-3510

\title{
Growth Models with Oblique Asymptote
}

\section{François Dubeau and Youness Mir}

\author{
Université de Sherbrooke \\ 2500 Boulevard de l'Université, J1K 2R1 Sherbrooke (Qc), Canada \\ E-mail: francois.dubeau@usherbrooke.ca \\ E-mail(corresp.): youness.mir@usherbrooke.ca
}

Received March 14, 2012; revised February 18, 2013; published online April 1, 2013

\begin{abstract}
A class of smooth functions which can be used as regression models for modelling phenomena requiring an oblique asymptote is analyzed. These types of models were defined as a product of a linear function and some well known growth models. In addition to their increasing character with an oblique asymptote, the resulting models provide curves with a single inflection point.
\end{abstract}

Keywords: growth models, oblique asymptote, single inflection point.

AMS Subject Classification: 26A48; 65D10; 62J02.

\section{Introduction}

Growth curve modelling using functions with an oblique asymptote and one or several inflection points are of interest in practice. Indeed, in economy smooth convex increasing curves with an oblique asymptote were considered in [30] to establish a relationship between household income and expenditure. In hydrology, for fitting the rating curves, it could be useful to have growth functions with one or more than one inflection point (see the U.S. Geological Survey's National Streamflow Information Program web page for more details). Also modelling with a varying carrying capacity leads to growth curve with more than one inflection point (see $[16,17,29]$ ).

In various fields, growth functions with an horizontal asymptote, such as Michaelis-Menten, Richards, Gompertz, Logistic, and Bridge functions, are commonly and widely used, e.g. agriculture $[9,10,11,12,18]$, biology $[1,8,19,20$, 31 ], economy [27], engineering [26], fishery [25], forestry [22,33,35], hydrology [2, $3,5,6,7,13,21,32]$, medicine [15], and other areas of applied research [23,24,28]. These growth functions are characterized by an horizontal asymptote and a single inflection point [4]. Other growth functions with these characteristics can be found in the literature (see $[9,14,34]$ for example).

In this paper we extend our previous study about the already mentioned models $[4,5,6]$. We modify them to obtain models with an oblique asymptote while keeping the single inflection point property. The structure of the present 
paper is as follows. In Section 2 we start with a general study of our modified model and obtain its main properties. In Section 3, using the analysis done in the preceding section, we modify some growth functions having a horizontal asymptote to obtain models having an increasing linear asymptote. Finally in Section 4 we present some numerical examples on synthetic data.

\section{Main Results}

Let us start with a general growth model $f(t)$, defined and twice continuously differentiable on an interval $\left(T_{0},+\infty\right)$, and having the following basic properties:

- $f(t) \geq 0$ is an increasing function on $\left(T_{0},+\infty\right)$,

- $f$ has one and only one inflection point on $\left(T_{0},+\infty\right)$ at $\left(t^{*}, f^{*}=f\left(t^{*}\right)\right)$,

- $\lim _{t \rightarrow T_{0}^{+}} f(t)=f_{T_{0}^{+}} \geq 0, \quad \lim _{t \rightarrow+\infty} f(t)=f_{+\infty}>f_{T_{0}^{+}}$.

Let us assume that the second derivative of this growth functions can be decomposed as follows

$$
f^{(2)}(t)=h(t) f^{(1)}(t)
$$

where

$$
\begin{aligned}
& f^{(1)}(t)>0 \text { for all } T_{0}<t<+\infty \\
& h(t) \begin{cases}>0 & \text { if } T_{0}<t<t^{*} \\
=0 & \text { if } t=t^{*} \\
<0 & \text { if } t>t^{*}\end{cases}
\end{aligned}
$$

Remember that the Michaelis-Menten, Richards (generalized Logistic), Gompertz, Logistic, and Bridge models given in Table 1 have these properties, see $[4]$.

In order to realize our goal of obtaining a growth function with an oblique asymptote, we multiply the growth function $f(t)$ by a linear increasing function given by $m(t)=p t+q$ with $p>0$ and $q \geq 0$ to obtain

$$
F(t)=m(t) f(t)
$$

Moreover the zero of $m(t)$, given by $-q / p$ is required to be much less than $t^{*}$, the abscissa of the inflection point of $f(t)$, then $-q / p<t^{*}$. We are going to consider $F(t)$ for $t>\max \left\{-q / p, T_{0}\right\}=\bar{T}_{0}$. Then we have

$$
F(t)=m(t) f(t)=[p t+q] f(t) \geq 0, \quad F^{(1)}(t)=p f(t)+m(t) f^{(1)}(t)>0 .
$$

Hence $F(t)$ is a positive increasing function for $t>\bar{T}_{0}$. Also, from (2.1),

$$
F^{(2)}(t)=2 p f^{(1)}(t)+m(t) f^{(2)}(t)=[2 p+h(t)(p t+q)] f^{(1)}(t),
$$

and it follows that

$$
F^{(2)}(t)>0 \text { for all } \bar{T}_{0}<t \leq t^{*} .
$$


Table 1. Parameter values and inflection point of the growth models.

\begin{tabular}{lclc}
\hline Model & Function & \multicolumn{1}{c}{ Parameters } & Inflection point $\left(t^{*}, f^{*}\right)$ \\
\hline Menten & $f_{M}(t)=\frac{\omega_{0} k^{c}+\omega_{f} t^{c}}{k^{c}+t^{c}}$ & $\begin{array}{l}k>0, c>1, \\
\omega_{f}>\omega_{0} \geq 0\end{array}$ & $\left(k\left(\frac{c-1}{c+1}\right)^{1 / c}, \frac{\omega_{0}+\omega_{f}}{2}-\frac{\omega_{f}-\omega_{0}}{2 c}\right)$ \\
Richards ${ }^{(1)}$ & $f_{R}(t)=a\left(1-b e^{-c t}\right)^{m}$ & $\begin{array}{l}a>0, b>0, \\
c>0, m>1\end{array}$ & $\left(\frac{\ln (m b)}{c}, a\left(1-\frac{1}{m}\right)^{m}\right)$ \\
Richards ${ }^{(2)}$ & $f_{R}(t)=a\left(1-b e^{-c t}\right)^{m}$ & $\begin{array}{l}a>0, b<0, \\
c>0, m<0\end{array}$ & $\left(\frac{\ln (m b)}{c}, a\left(1-\frac{1}{m}\right)^{m}\right)$ \\
Gompertz & $f_{G}(t)=a e^{-b e^{-c t}}$ & $a>0, b>0, c>0$ & $\left(\frac{\ln (b)}{c}, a e^{-1}\right)$ \\
Logistic & $f_{L}(t)=\frac{a}{1+b e^{-c t}}$ & $a>0, b>0, c>0$ & $\left(\frac{\ln (b)}{c}, \frac{a}{2}\right)$ \\
Bridge & $f_{B}(t)=a\left(1-e^{-m t^{b}}\right)$ & $a>0, b>1, m>0$ & $\left(\left(\frac{b-1}{m b}\right)^{1 / b}, a\left(1-e^{-\frac{b-1}{b}}\right)\right)$ \\
\hline
\end{tabular}

Moreover, suppose we can write

$$
2 p+h(t)(p t+q)=Q(t) g(t)
$$

where $g(t)>0$ for $t \in\left(\bar{T}_{0},+\infty\right)$. Then $Q(t)>0$ for $t \in\left(\bar{T}_{0}, t^{*}\right]$, and

$$
Q\left(t^{*}\right)=\frac{2 p}{g\left(t^{*}\right)}>0 .
$$

Since we have

$$
F^{(2)}(t)=Q(t) g(t) f^{(1)}(t)
$$

to show that $F(t)$ has one and only one inflection point, it remains to prove that the sign of $Q(t)$ changes only once on $\left(t^{*},+\infty\right)$. Some conditions on the expression of $Q(t)$ which ensure this fact are given in the next two theorems.

Theorem 1. Let $Q(t)$ be a function defined by

$$
Q(t)=(A+B t)-(C+D t) t^{\gamma} \quad \text { for } t \geq 0
$$

where $B>0$ and $D>0$ are two positive real numbers and $\gamma \geq 1$. If there exists a positive real number $t^{*} \geq 0$ such that $Q\left(t^{*}\right) \geq 0$, then there exists a unique $T^{*}>t^{*}$ such that

$$
Q(t) \begin{cases}>0 & \text { if } t^{*} \leq t<T^{*}, \\ =0 & \text { if } t=T^{*} \\ <0 & \text { if } t>T^{*}\end{cases}
$$

Proof. Let us suppose that there exists a $t^{*} \geq 0$ such that $Q\left(t^{*}\right)>0$. Under the assumptions on $D$ and $\gamma$, we also have

$$
\lim _{t \rightarrow+\infty} Q(t)=-\infty
$$


For the first derivative of $Q(t)$, we have

$$
\begin{aligned}
Q^{(1)}(t) & =B-[D(\gamma+1) t+\gamma C] t^{\gamma-1} \\
& =\left\{\frac{1}{t^{\gamma-1}}-\left[\frac{D(\gamma+1) t}{B}+\frac{\gamma C}{B}\right]\right\} B t^{\gamma-1} .
\end{aligned}
$$

In the above equation the linear factor in brackets is strictly increasing since $B$ and $D$ are strictly positive. So to determine the sign of the term between braces we have to study two cases.

Case $\gamma>1$ : the function $t \mapsto 1 / t^{\gamma-1}$ is strictly decreasing, so there exists a unique $\tau>0$ such that $Q^{(1)}(\tau)=0$, and then

$$
Q^{(1)}(t) \begin{cases}>0 & \text { if } 0<t<\tau \\ =0 & \text { if } t=\tau \\ <0 & \text { if } t>\tau\end{cases}
$$

Case $\gamma=1$ : the existence of $\tau>0$ such that $Q^{(1)}(\tau)=0$ requires that $C<B$, otherwise we have $Q^{(1)}(t)<0$ for all $t>0$.

In both cases above, there exists a unique $T^{*}>t^{*}$ such that the condition (2.4) holds.

Remark. We can allow $D=0$ in Theorem 1 and the conclusion holds under the following conditions :

- If $\gamma=1$, we have $Q(t)=A+(B-C) t$. In this case, to ensure that the condition (2.4) holds we must have $B<C$, because otherwise for all $t \geq t^{*}$ we have $Q(t) \geq Q\left(t^{*}\right)>0$.

- If $\gamma>1$, we must have $C>0$ because otherwise $Q^{(1)}(t)>0$ for all $t \geq t^{*}$.

Theorem 2. Let $Q(t)$ be a function defined by

$$
Q(t)=(A-B t)+(C+D t) e^{-\gamma t} \quad \text { for } t \in \mathbb{R},
$$

where $B>0$ and $D \geq 0$ are two positive real numbers and $\gamma>0$. Suppose there exists a real number $t^{*}$ such that $Q\left(t^{*}\right)>0$, then there exists a unique $T^{*}>t^{*}$ such that

$$
Q(t) \begin{cases}>0 & \text { if } t^{*} \leq t<T^{*}, \\ =0 & \text { if } t=T^{*} \\ <0 & \text { if } t>T^{*}\end{cases}
$$

Proof. Let us assume that there exists $t^{*} \in \mathbb{R}$ such that $Q\left(t^{*}\right)>0$. Under the assumptions on $B$ and $\gamma$ we also have

$$
\lim _{t \rightarrow+\infty} Q(t)=-\infty
$$

For the first derivative of $Q(t)$, we have

$$
\begin{aligned}
Q^{(1)}(t) & =-B+[D-\gamma(C+D t)] e^{-\gamma t} \\
& =\left\{-e^{\gamma t}+\left[\frac{D-\gamma C}{B}-\frac{\gamma D}{B} t\right]\right\} B e^{-\gamma t} .
\end{aligned}
$$


In the above equation the linear factor in brackets is strictly decreasing since $B, D$, and $\gamma$ are strictly positive (or a constant if $D=0$ ). Moreover, the exponential function $t \mapsto e^{\gamma t}$ is strictly increasing with $\lim _{t \rightarrow-\infty} e^{\gamma t}=0$ and $\lim _{t \rightarrow+\infty} e^{\gamma t}=+\infty$. Thus, there exists one and only one $\tau \in \mathbb{R}$ such that $Q^{(1)}(\tau)=0$ and then

$$
Q^{(1)}(t) \begin{cases}>0 & \text { if } t<\tau \\ =0 & \text { if } t=\tau \\ <0 & \text { if } t>\tau .\end{cases}
$$

Hence the function $Q(t)$ is strictly increasing for $t \leq \tau$ and strictly decreasing for $t \geq \tau$. Since $Q\left(t^{*}\right)>0$, there exists a unique $T^{*}>t^{*}$ such that the condition (2.5) holds.

From the two theorems, we have

$$
F^{(2)}(t) \begin{cases}>0 & \text { if } \bar{T}_{0}<t<T^{*} \\ =0 & \text { if } t=T^{*} \\ <0 & \text { if } t>T^{*}\end{cases}
$$

The function $F(t)$ is a positive strictly increasing function, convex on $\left(\bar{T}_{0}, T^{*}\right)$, concave on $\left(T^{*},+\infty\right)$, with a single inflection point at $\left(T^{*}, F^{*}=F\left(T^{*}\right)\right)$. Moreover the oblique asymptote is given by $y=[p t+q] f_{+\infty}$.

In the next section, we apply these previous ideas on the six models mentioned in Table 1. This study does not cover all the existing models used in practice (see $[11,12,14,23,34,35]$ ), but any other model with a sigmoidal behavior can be modified in a similar way with the use of Theorem 1 or Theorem 2 .

\section{Models}

The basic models are given in Table 1 . We use them and consider the product

$$
F(t)=m(t) f(t)=[p t+q] f(t)
$$

to obtain the modified models listed in Table 2. Let us observe that the multiplicative constants, $\omega_{f}$ or $a$, in the basic models are included in the parameters $p$ and $q$ of the modified models, and $f_{+\infty}=1$. Using the decomposition (2.3), we look for a unique $T^{*}>t^{*}$ such that

$$
Q(t) \begin{cases}>0 & \text { if } t^{*} \leq t<T^{*} \\ =0 & \text { if } t=T^{*} \\ <0 & \text { if } t>T^{*}\end{cases}
$$

to obtain the desired shape for the graph of $F(t)$ for the values of the parameters $(p, q, \theta)$ in the feasible set $\Theta$ specified in the last two columns of the Table 2 . The graphs of $f$ and $F$ for all these models look like the graphs given in Figure 1. 
Table 2. Growth models with an oblique asymptote $y=p t+q$.

\begin{tabular}{llcc}
\hline Model & \multicolumn{1}{c}{ Function } & \multicolumn{2}{c}{ Parameters $(p, q, \theta) \in \Theta$} \\
\cline { 2 - 4 } & $F(t)=[p t+q] f(t)$ & $p, q$ & $\theta$ \\
\hline Michaelis-Menten & $F_{M}(t)=[p t+q] \frac{\omega_{0} k^{c}+t^{c}}{k^{c}+t^{c}}$ & $p>0, q \geq 0$ & $k>0, c>1,0 \leq \omega_{0}<1$ \\
Richards $^{(1)}$ & $F_{R}(t)=[p t+q]\left(1-b e^{-c t}\right)^{m}$ & $p>0, q \geq 0$ & $b>0, c>0, m>1$ \\
Richards $^{(2)}$ & $F_{R}(t)=[p t+q]\left(1-b e^{-c t}\right)^{m}$ & $p>0, q \geq 0$ & $b<0, c>0, m<0$ \\
Gompertz & $F_{G}(t)=[p t+q] e^{-b e^{-c t}}$ & $p>0, q \geq 0$ & $b>0, c>0$ \\
Logistic & $F_{L}(t)=\frac{[p t+q]}{1+b e^{-c t}}$ & $p>0, q \geq 0$ & $b>0, c>0$ \\
Bridge & $F_{B}(t)=[p t+q]\left(1-e^{-m t^{b}}\right)$ & $p>0, q \geq 0$ & $b>1, m>0$ \\
\hline
\end{tabular}

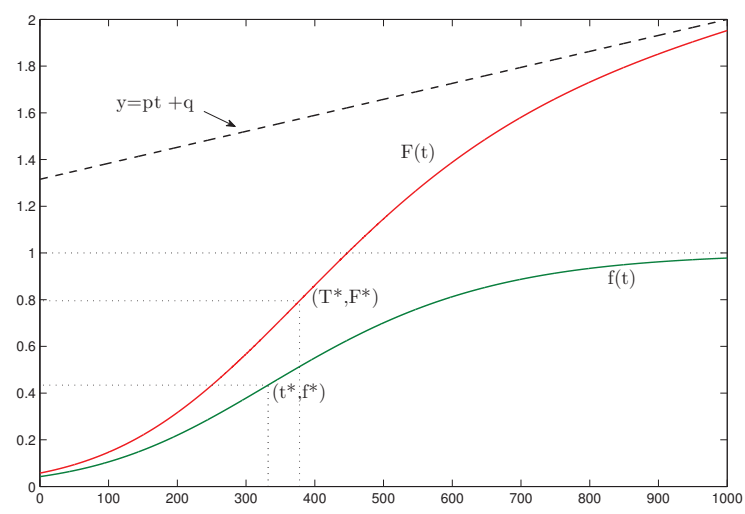

Figure 1. Graphic representation of a growth model $f(t)$ and its modified model $F(t)$ with their respective asymptotes $y=1$ and $y=p t+q$ and inflection points $\left(t^{*}, f^{*}\right)$ and $\left(T^{*}, F^{*}\right)$.

\subsection{Michaelis-Menten model $f_{M}(t)=\left(\omega_{0} k^{c}+t^{c}\right) /\left(k^{c}+t^{c}\right)$}

To ensure the increasing character of this function with the existence of an inflection point we assume that $k>0, c \geq 1$ and $0 \leq \omega_{0}<1$. The MichaelisMenten function is well defined when $t \geq T_{0}=0$. Moreover we have

$$
f_{M}^{(1)}(t)=\frac{c\left(1-\omega_{0}\right) k^{c} t^{c-1}}{\left(k^{c}+t^{c}\right)^{2}} \quad \text { and } \quad f_{M}^{(2)}(t)=\left[(c-1) k^{c}-(c+1) t^{c}\right] \frac{f_{M}^{(1)}(t)}{t\left(k^{c}+t^{c}\right)} .
$$

From (3.1) and (2.2), it follows that

$$
F_{M}^{(2)}(t)=\left[k^{c}(q(c-1)+p(c+1) t)-(q(c+1)+p(c-1) t) t^{c}\right] \frac{f_{M}^{(1)}(t)}{t\left(k^{c}+t^{c}\right)} .
$$

Then we have

$$
Q(t)=k^{c}(q(c-1)+p(c+1) t)-(q(c+1)+p(c-1) t) t^{c},
$$


and

$$
g(t)=\frac{1}{t\left(k^{c}+t^{c}\right)}
$$

Thus, Theorem 1 with $B=(c+1) p>0, D=(c-1) p \geq 0$ and $\gamma=c$ ensures the existence of a unique $T^{*}>t^{*}$ such that the condition (3.2) holds.

\subsection{Richards model $f_{R}(t)=\left(1-b e^{-c t}\right)^{m}$}

The function is defined as long as $1-b e^{-c t} \geq 0$. In this case we have

$$
f_{R}^{(1)}(t)=\frac{m b c e^{-c t}}{1-b e^{-c t}} f_{R}(t) \quad \text { and } \quad f_{R}^{(2)}(t)=-c \frac{1-m b e^{-c t}}{1-b e^{-c t}} f_{R}^{(1)}(t) .
$$

From (2.2) and (3.1), we can write

$$
F_{R}^{(2)}(t)=\left[((2 p-c q)-c p t)+((m b c q-2 b p)+b c m p t) e^{-c t}\right] \frac{f_{R}^{(1)}(t)}{1-b e^{-c t}} .
$$

It follows that

$$
Q(t)=((2 p-c q)-c p t)+((m b c q-2 b p)+b c m p t) e^{-c t}
$$

and

$$
g(t)=\frac{1}{1-b e^{-c t}} .
$$

Two sets of conditions on the parameters are allowed here to ensure the increasing character of Richards function with the existence of an inflection point.

First case: $\operatorname{Richards}^{(1)}$ with $a>0, b>0, c>0, m>1$, and $T_{0}=\frac{\ln (b)}{c}$.

Second case: Richards ${ }^{(2)}$ with $a>0, b<0, c>0, m<0$, and $T_{0}=-\infty$.

Then, Theorem 2 with $B=c p, D=c m b p>0$ and $\gamma=c>0$ ensures in both cases the existence of a unique $T^{*}>t^{*}$ such that the condition (3.2) holds.

\subsection{Gompertz model $f_{G}(t)=e^{-b e^{-c t}}$}

It is well known that the Gompertz model is a limiting case of Richards ${ }^{(1)}$ model for $m_{\mathrm{R}} \rightarrow+\infty, b_{\mathrm{R}} \rightarrow 0^{+}$, and $m_{\mathrm{R}} b_{\mathrm{R}} \rightarrow b_{\mathrm{G}}$, and of Richards ${ }^{(2)}$ model for $m_{\mathrm{R}} \rightarrow-\infty, b_{\mathrm{R}} \rightarrow 0^{-}$, and $m_{\mathrm{R}} b_{\mathrm{R}} \rightarrow b_{\mathrm{G}}$. This function is well defined on $\mathbb{R}$ and $T_{0}=-\infty$. It is a strictly increasing function with one inflection point for $b>0$ and $c>0$. We have

$$
f_{G}^{(1)}(t)=b c e^{-c t} f_{G}(t) \quad \text { and } \quad f_{G}^{(2)}(t)=-c\left[1-b e^{-c t}\right] f_{G}^{(1)}(t) .
$$

Hence, from (2.2) and (3.1), we get

$$
F_{G}^{(2)}(t)=\left[((2 p-c q)-c p t)+(b c q+b c p t) e^{-c t}\right] f_{G}^{(1)}(t),
$$

then

$$
Q(t)=((2 p-c q)-c p t)+(b c q+b c p t) e^{-c t},
$$

and $g(t)=1$. Thus, Theorem 2 with $B=c p, D=b c p$ and $\alpha=c>0$ provides that there exists a unique $T^{*}>t^{*}$ such that the condition (3.2) holds. 


\subsection{Logistic model $f_{L}(t)=\frac{1}{1+b e^{-c t}}$}

Obviously, the Logistic model is a particular case of the Richards ${ }^{(2)}$ model with the parameter $m$ equal to -1 and $b$ is replaced by $-b$. It is a well defined function on $\mathbb{R}$ and $T_{0}=-\infty$. We must have $b>0$ and $c>0$ to have one inflection point at $t^{*}=\frac{\ln (b)}{c}$. Since

$$
f_{L}^{(1)}(t)=\frac{b c e^{-c t}}{1+b e^{-c t}} f_{L}(t)=c f_{L}(t)\left[1-f_{L}(t)\right]
$$

and

$$
f_{L}^{(2)}(t)=-\frac{c\left(1-b e^{-c t}\right)}{1+b e^{-c t}} f_{L}^{(1)}(t)=c\left[1-2 f_{L}(t)\right] f_{L}^{(1)}(t)
$$

Hence, from (2.2) and (3.1), it follows that

$$
F_{L}^{(2)}(t)=\left[((2 p-c q)-c p t)+(b(2 p+c q+b c p t)) e^{-c t}\right] \frac{f_{L}^{(1)}(t)}{1+b e^{-c t}}
$$

Hence we set

$$
Q(t)=((2 p-c q)-c p t)+(b(2 p+c q+b c p t)) e^{-c t}
$$

and

$$
g(t)=\frac{1}{1+b e^{-c t}}
$$

From Theorem 2 with $B=c p, D=b c p$, and $\alpha=c>0$ we have the existence of a unique $T^{*}>t^{*}$ such that the condition (3.2) holds.

3.5 Bridge model $f_{B}(t)=1-e^{-m t^{b}}$

The Bridge function is well defined when $t \geq T_{0}=0$. Moreover, we assume that $b \geq 1$ and $m>0$ to have an increasing function with one inflection point. We have

$$
f_{B}^{(1)}(t)=m b t^{b-1} e^{-m t^{b}} \quad \text { and } \quad f_{B}^{(2)}(t)=\frac{(b-1)-m b t^{b}}{t} f_{B}^{(1)}(t) .
$$

From (3.1) and (2.2), we obtain

$$
F_{B}^{(2)}(t)=\left[(q(b-1)+(b+1) p t)-(m b q+m b p t) t^{b}\right] \frac{f_{B}^{(1)}(t)}{t} .
$$

Then we have

$$
Q(t)=(q(b-1)+(b+1) p t)-m b(q+p t) t^{b}, \quad g(t)=\frac{1}{t} .
$$

Thus Theorem 1 with $B=(b+1) p>0, D=m b p>0$ and $\gamma=b>1$ ensures the existence of a unique $T^{*}>t^{*}$ such that the condition (3.2) holds. 


\section{Numerical Examples}

In this section we use the six models presented in the last section to fit some synthetic data sets $\left\{\left(t_{k}, y_{k}\right)\right\}_{k=1}^{K}$. Because the numerical behavior of the original models of Table 1 has been studied elsewhere, in this section we focus on the asymptote of the modified models of Table 2. Firstly, we compare the values of the two parameters of the asymptote, the slope $p$ and the $y$-intercept $q$, computed from the fitting of the six models on the same data sets. Because a comparison of the models based on their best fits depends widely on the data set, we briefly mention the quality of the fitting, we compare and rank the fitting of the models using the value of the optimal criteria. Secondly, for a given model, the Richards ${ }^{(2)}$ model, we illustrate the effect of increasing the density of data to recover the values of the parameters $p$ and $q$ of the asymptote.

\subsection{Numerical computation}

The parameter vector $(p, q, \theta)$ of each model $F(t)$ is estimated by minimizing the least squares criteria

$$
Z(p, q, \theta)=\frac{1}{2} \sum_{k=1}^{K}\left[F\left(t_{k} ;(p, q, \theta)\right)-y_{k}\right]^{2}
$$

over the feasible set of parameters $\Theta$ as defined for each model in the last two columns of Table 2. We used the MATLAB least squares function "lsqnonlin" to solve these problems. The MATLAB function "fsolve" was used to solve the equation $Q(t)=0$ to obtain an approximation $T^{* *}$ of the abscissa $T^{*}$ of the inflection point $\left(T^{*}, F^{*}\right)$ for each modified model, and the approximate ordinate $F^{* *}$ is given by $F^{* *}=F\left(T^{* *}\right)$. Numerical results of our simulations include the optimal solution $\left(p^{*}, q^{*}, \theta^{*}\right)$, the optimal value of the criteria $Z^{*}$, its mean optimal value $Z^{*} / K=Z\left(p^{*}, q^{*}, \theta^{*}\right) / K$, and an approximation $\left(T^{* *}, F^{* *}\right)$ of the inflection point $\left(T^{*}, F^{*}\right)$.

\subsection{First example}

\subsubsection{Data sets}

To generate the first series of data, we have first chosen the exponential model $F_{E}(t ; p, q, \theta)=(p t+q) e^{-\alpha / t^{\gamma}}$, where $\theta=(\alpha, \gamma)$, because the shape of its graph looks like the graph of the models under study. We have used the value $(p, q, \theta)=(0.5,100,3000,1.4)$ for its parameters. We have generated 10 random uniformly distributed abscissae on the interval $(0,2000]$, by choosing $t_{k} \sim \mathcal{U}(0,2000]$, and 10 random uniformly distributed abscissae on the (2000, 4000], by choosing $t_{k} \sim \mathcal{U}(2000,4000]$. Then the ordinates are computed by $y_{k}=F_{E}\left(t_{k} ; p, q, \theta\right)+r_{k}$ for $k=1, \ldots, 20$, where $r_{k} \sim \mathcal{N}\left(0, \sigma^{2}\right)$ with $\sigma=10$.

The six models of Table 2 were then fitted to the 20 data on $(0,4000]$. With each of these optimal models we have generated 20 other data points, ten on each subintervals $(0,2000]$ and $(2000,4000]$, as we did before with the exponential model. We have added together these data points to get two sets 
of 70 data points on each intervals $(0,2000]$ and $(2000,4000]$. In this way we can say that the data represent all the models.

We have considered the best fitting for the following situations:

- on the interval $(0,2000]$ with 70 data points;

- on the interval $(0,4000]$ with 140 data points;

- on the interval $(0,4000]$ with 70 data points (chosen randomly in the preceding data set with 35 data points on each subinterval $(0,2000]$ and $(2000,4000])$;

The results are reported in Table 3 . For each data set, and on each model $F$, we have tested 10 different starting points $\left(p_{0}, q_{0}, \theta_{0}\right)$ randomly chosen in the feasible set $\Theta$ using continuous uniform distributions. For each model and each data set, when the starting values are chosen around the values of the parameters used to generate the data, the algorithm has always converged to the same optimal solution from any one of these starting points.

\subsubsection{Numerical results and discussion}

From Table 3 we observe that the asymptotes recovered by the Gompertz, Logistic, Richards ${ }^{(1)}$, Richards ${ }^{(2)}$, and Bridge models have the slope $p^{*}$ and y-intercept $q^{*}$ relatively close together. These models recover almost the same asymptote. For the Michaelis-Menten model the slope $p^{*}$ is also close to the values obtained by the preceding 5 models, but the y-intercept $q^{*}$ is slightly bigger. This differences in $q^{*}$ decreases if we increase the length $T$ of the interval, from 2000 to 4000 , maintaining the same density $K / T$ of data as shown when we compare the parameter values of Table 3.

Based on the optimal value of the criteria $Z^{*} / K$, the numerical results summarized in Table 4 indicates that the best fit is obtained for the Richards ${ }^{(2)}$ model followed by the Bridge model, but the differences between the $Z^{*} / K$ for the 6 models are rather small. We also observe the same results for the Richards ${ }^{(1)}$ and the Gompertz models because $m_{R} b_{R} \approx b_{G}$. Obviously, the rank for the models might change depending on the data set under consideration. We have included, in Figures 2 and 3, the graphs of the best model, the Richards ${ }^{(2)}$ model, and the graphs of the worst one, the Michaelis-Menten model, on the intervals $(0,2000]$ and $(0,4000]$ to illustrate that globally the fitting is good for all models. We can see slight differences on the graphs of the asymptotes. Let us observe that when we increase the length $T$ with the same data density, the mean value $Z^{*} / K$ decreases, which means we get more information for the fitting and obtain a more reliable asymptote when $T$ is large.

\subsection{Second example}

\subsubsection{Data sets}

In this section we have tested Richards ${ }^{(2)}$ on different data sets $\left\{\left(t_{k}, y_{k}\right)\right\}_{k=1}^{K}$ with different values of $K$ on the interval $(0,4000]$. The data points $\left\{\left(t_{k}, y_{k}\right)\right\}_{k=1}^{K}$ 
Table 3. Optimal parameters, optimal value and an approximation of the inflection point $\left(T^{* *}, F^{* *}\right)$ for each data set $(K, T)$.

\begin{tabular}{|c|c|c|c|c|c|c|c|c|}
\hline \multicolumn{9}{|c|}{ Michealis-Menten model: } \\
\hline$K$ & $T$ & $p^{*}$ & $q^{*}$ & $\omega_{0}^{*}$ & $k^{*}$ & $c^{*}$ & $Z^{*}$ & $\left(T^{* *}, F^{* *}\right)$ \\
\hline 70 & 2000 & 0.4933 & 142.04 & 0.09506 & 403.34 & 2.9780 & 3840.4 & $(420.78,201.36)$ \\
\hline 140 & 4000 & 0.5102 & 106.45 & 0.15396 & 393.03 & 3.4883 & 6820.6 & $(415.07,196.38)$ \\
\hline 70 & 4000 & 0.5117 & 102.53 & 0.16045 & 398.77 & 3.5697 & 3203.3 & $(421.75,198.02)$ \\
\hline$T$ & $K$ & $p^{*}$ & $q^{*}$ & $b^{*}$ & $c^{*}$ & $m^{*}$ & $Z^{*}$ & $\left(T^{* *}, F^{* *}\right)$ \\
\hline \multicolumn{9}{|c|}{$\operatorname{Richards}^{(1)}$ model: $\quad F_{R}(t ; p, q, b, c, m)$} \\
\hline 70 & 2000 & 0.5090 & 103.28 & 0.00038 & 0.00536 & 11379 & 3752.5 & $(436.83,214.60)$ \\
\hline 140 & 4000 & 0.5128 & 96.957 & 0.00047 & 0.00551 & 9447.4 & 6587.9 & $(430.23,209.89)$ \\
\hline 70 & 4000 & 0.5136 & 95.341 & 0.00036 & 0.00540 & 12032 & 3115.8 & $(438.99,213.49)$ \\
\hline \multicolumn{9}{|c|}{$\operatorname{Richards}^{(2)}$ model: $\quad F_{R}(t ; p, q, b, c, m)$} \\
\hline 70 & 2000 & 0.5155 & 91.963 & -3.1315 & 0.00631 & -2.1955 & 3709.9 & $(449.43,223.57)$ \\
\hline 140 & 4000 & 0.5137 & 94.305 & -2.8042 & 0.00622 & -2.3696 & 6470.6 & $(449.68,223.76)$ \\
\hline 70 & 4000 & 0.5148 & 91.792 & -4.3383 & 0.00639 & -1.7672 & 3068.2 & $(462.23,229.84)$ \\
\hline \multicolumn{9}{|c|}{ Gompertz model: $\quad F_{G}(t ; p, q, b, c)$} \\
\hline 70 & 2000 & 0.5090 & 103.28 & 4.3372 & 0.00536 & & 3752.4 & $(436.83,214.60)$ \\
\hline 140 & 4000 & 0.5128 & 96.956 & 4.4386 & 0.00551 & & 6587.8 & $(430.24,209.89)$ \\
\hline 70 & 4000 & 0.5136 & 95.341 & 4.3621 & 0.00540 & & 3115.8 & $(438.99,213.49)$ \\
\hline \multicolumn{9}{|c|}{ Logistic model: $\quad F_{L}(t ; p, q, b, c)$} \\
\hline 70 & 2000 & 0.5206 & 80.347 & 11.916 & 0.00740 & & 3777.8 & $(460.61,236.87)$ \\
\hline 140 & 4000 & 0.5146 & 91.473 & 11.711 & 0.00721 & & 6603.4 & $(468.52,237.67)$ \\
\hline 70 & 4000 & 0.5155 & 89.611 & 11.964 & 0.00716 & & 3110.1 & $(474.90,239.16)$ \\
\hline \multicolumn{9}{|c|}{ Bridge model: $\quad F_{B}(t ; p, q, b, m)$} \\
\hline 70 & 2000 & 0.5189 & 87.746 & 1.7673 & & $2.473 e-5$ & 3744.3 & $(452.40,234.05)$ \\
\hline 140 & 4000 & 0.5144 & 92.235 & 1.7492 & & $2.679 e-5$ & 6528.3 & $(467.86,237.98)$ \\
\hline 70 & 4000 & 0.5151 & 90.758 & 1.7476 & & $2.642 e-5$ & 3096.6 & $(476.07,240.97)$ \\
\hline
\end{tabular}

Table 4. Ranking criterion values for the models.

\begin{tabular}{|c|c|c|c|c|c|c|}
\hline \multirow[b]{2}{*}{ Model } & \multicolumn{3}{|c|}{$\begin{array}{c}\text { Data set } \\
K=70 \text { and } T=2000\end{array}$} & \multicolumn{3}{|c|}{$\begin{array}{c}\text { Data set } \\
K=140 \text { and } T=4000\end{array}$} \\
\hline & $Z^{*}$ & $Z^{*} / K$ & Rank & $Z^{*}$ & $Z^{*} / K$ & Rank \\
\hline Michaelis-Menten & 3840.4 & 54.863 & 6 & 6820.6 & 48.719 & 6 \\
\hline Richards $^{(1)}$ & 3752.4 & 53.606 & 3 & 6587.8 & 47.056 & 3 \\
\hline $\operatorname{Richards}^{(2)}$ & 3709.9 & 52.999 & 1 & 6470.6 & 46.219 & 1 \\
\hline Gompertz & 3752.4 & 53.606 & 3 & 6587.8 & 47.056 & 3 \\
\hline Logistic & 3777.8 & 53.969 & 5 & 6603.4 & 47.167 & 5 \\
\hline Bridge & 3744.3 & 53.490 & 2 & 6528.3 & 46.631 & 2 \\
\hline
\end{tabular}

are generated, for $k=1, \ldots, K$, by

$$
y_{k}=\left(p t_{k}+q\right) F_{R}\left(t_{k} ; \theta\right)+r_{k},
$$




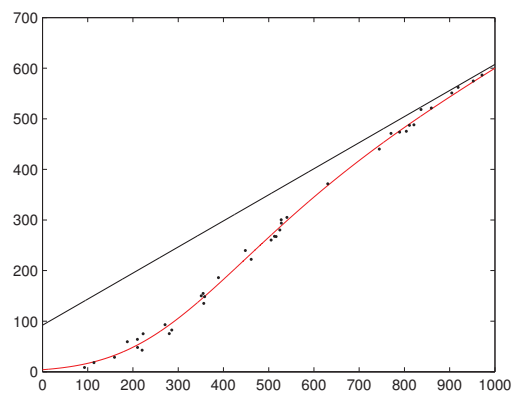

(a) $(K=70, T=2000)$

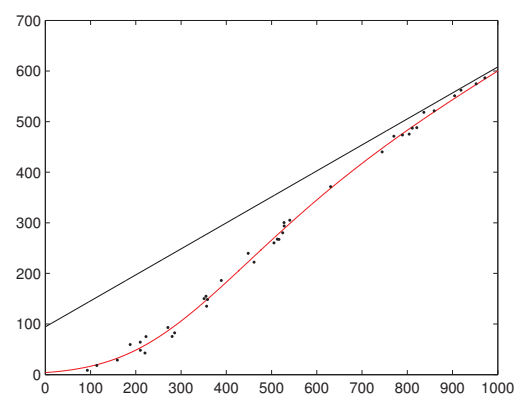

(b) $(K=140, T=4000)$

Figure 2. Graphic representation of the synthetic data points $\left\{t_{k}, y_{k}\right\}_{k=1}^{K}$ with the modified Richards ${ }^{(2)}$ model $F_{R}(t ; p, q, \theta)=(p t+q) f_{R}(t ; \theta)$ and its asymptote at the optimal solution $\left(p^{*}, q^{*}, \theta^{*}\right)$.

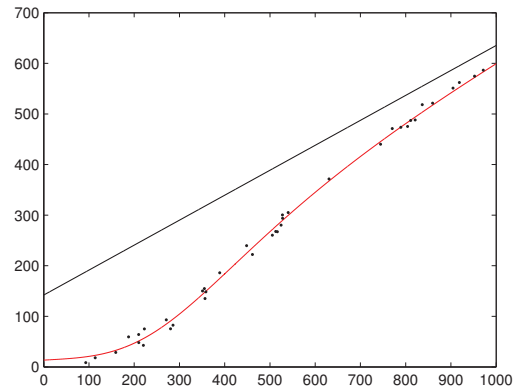

(a) $(K=70, T=2000)$

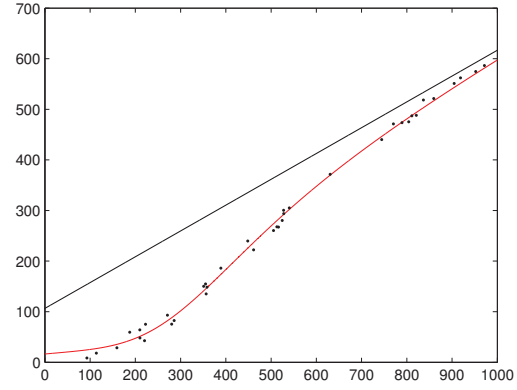

(b) $(K=140, T=4000)$

Figure 3. Graphic representation of the synthetic data points $\left\{t_{k}, y_{k}\right\}_{k=1}^{K}$ with the modified Michaelis-Menten model $F_{M}(t ; p, q, \theta)=(p t+q) f_{M}(t ; \theta)$ and its asymptote $m(t)=p t+q$ at the optimal solution $\left(p^{*}, q^{*}, \theta^{*}\right)$.

where $(p, q, \theta)=(0.5,100,-3,0.005,-2), t_{k} \sim \mathcal{U}(0,4000)$ is a uniformly distributed random variable over $(0, T], r_{k} \sim \mathcal{N}\left(0, \sigma^{2}\right)$ is a random normal variable with mean $\mu=0$ and standard deviation $\sigma=4$. Numerical results are summarized in Table 5 .

\subsubsection{Numerical results and discussion}

The results of the Table 5 indicate a monotonic convergence of the estimated values of the parameters through the true values of the parameters as the density $K$ of the data increases in the interval.

\section{Conclusion}

In this paper we have extended a class of standard growth curves with horizontal asymptote in order to obtain growth curves with linear asymptote. The 
Table 5. Richards ${ }^{(2)}$ model $(T=4000)$ : Optimal parameters, optimal value and an approximation of the inflection point $\left(T^{* *}, F^{* *}\right)$ for different data sets with an increasing degree of freedom.

\begin{tabular}{rcccccccc}
\hline \multicolumn{7}{c}{ Richards $^{(2)}$ model: } & $F_{R}(t ; p, q, b, c, m)$ & \\
\hline $\begin{array}{c}\text { True } \\
\text { values }\end{array}$ & $p$ & $q$ & $b$ & $c$ & $m$ & & & $\left(T^{*}, F^{* *}\right)$ \\
& 0.5 & 100 & -3 & 0.005 & -2 & & & $(555.9,268.63)$ \\
\hline$K$ & $p^{*}$ & $q^{*}$ & $b^{*}$ & $c^{*}$ & $m^{*}$ & $Z^{*}$ & $Z^{*} / K$ & $\left(T^{*}, F^{* *}\right)$ \\
\hline 20 & 0.5055 & 91.41 & -10.08 & 0.00574 & -0.7447 & 103.38 & 5.169 & $(594.6,300.33)$ \\
40 & 0.5053 & 92.52 & -6.706 & 0.00569 & -0.9774 & 306.57 & 7.664 & $(585.9,287.99)$ \\
60 & 0.5048 & 93.27 & -6.951 & 0.00566 & -1.1920 & 388.41 & 6.474 & $(571.1,280.08)$ \\
80 & 0.5047 & 93.31 & -5.708 & 0.00557 & -1.2013 & 452.86 & 5.661 & $(573.9,278.83)$ \\
100 & 0.5021 & 94.73 & -4.828 & 0.00543 & -1.3373 & 715.41 & 7.154 & $(561.8,270.79)$ \\
120 & 0.5013 & 96.69 & -4.688 & 0.00538 & -1.3561 & 772.29 & 6.436 & $(561.3,272.61)$ \\
140 & 0.5007 & 98.16 & -3.774 & 0.00538 & -1.5728 & 921.59 & 6.598 & $(558.5,266.42)$ \\
160 & 0.5007 & 98.54 & -3.661 & 0.00507 & -1.7069 & 1139.2 & 7.120 & $(558.8,266.47)$ \\
180 & 0.5005 & 98.89 & -3.177 & 0.00507 & -1.9521 & 1362.5 & 7.569 & $(559.6,271.94)$ \\
200 & 0.5000 & 99.89 & -2.924 & 0.00495 & -1.9814 & 1497.7 & 7.489 & $(557.3,270.24)$ \\
\hline
\end{tabular}

objective of the present study was to present such models and show their performances on synthetic data sets. In addition to their increasing character with an oblique asymptote, the resulting models provide curves with a single inflection point. Other extensions of these models to obtain curvilinear asymptote and growth curves with several inflection points could be of interest. These extensions and their applications will be presented in a future paper.

\section{Acknowledgments}

This work has been partially supported by a NSERC (Natural Sciences and Engineering Research Council of Canada) individual discovery grant for the first author.

\section{References}

[1] L. Von Bertalanffy. Quantitative laws in metabolism and growth. The Quarterly Review of Biology, 32:217-231, 1957. http://dx.doi.org/10.1086/401873.

[2] J.E. Bullard, G.H. Mctainsh and P. Martin. Establishing stage-discharge relationships in multiple-channelled, ephemeral rivers: a case study of the Diamantina River. Geographical Research, 45:233-245, 2007. http://dx.doi.org/10.1111/j.1745-5871.2007.00457.x.

[3] J. Callede, P. Kosuth and E. de Oliveira. Établissement de la relation hauteurdébit de l'Amazone à Obidos: méthode de la dénivelée normale à "géométrie variable". Hydrological Sci. J., 46:451-463, 2001. http://dx.doi.org/10.1080/02626660109492838.

[4] F. Dubeau and Y. Mir. Least squares fitting with single inflection point growth curve I - The models. Math. Model. Appl. Comput., 2:269-281, 2011. 
[5] F. Dubeau, Y. Mir, A.A. Assani and A. Chalifour. Least squares fitting with single inflection point growth curve II - An application. Math. Model. Appl. Comput., 2:283-301, 2011.

[6] F. Dubeau, Y. Mir, A.A. Assani and A. Chalifour. Modelling stage-discharge relationship with single inflection point nonlinear functions. Int. J. Hydrology Sci. Technol., 2:153-167, 2012. http://dx.doi.org/10.1504/IJHST.2012.047430.

[7] E.H. Habid and E.A. Meselhe. Stage-Discharge relations for low-gradient tidal streams using data-driver models. ASCE J. Hydraul. Eng., 132:482-492, 2006. http://dx.doi.org/10.1061/(ASCE)0733-9429(2006)132:5(482).

[8] S. Huet, E. Jolivet and A. Messéan. La régression non linéaire: méthodes et applications en biologie. INRA, Paris, 1992.

[9] Z. Ismail, A. Khamis and M.Y. Jaafar. Fitting nonlinear Gompertz curve to tobacco growth data. Pakistan J. Agronomy, 2:223-236, 2003. http://dx.doi.org/10.3923/ja.2003.223.236.

[10] R.C. Jain, R. Agrawal and K.N. Singh. A whitin year growth model for crop yield forecasting. Biom. J., 34:789-799, 1992. http://dx.doi.org/10.1002/bimj.4710340705.

[11] A. Khamis, Z. Ismail, K. Haron and A.T.M. Mohammed. Nonlinear growth models for modeling oil palm yield growth. J. Math. Stat., 1:225-233, 2005. http://dx.doi.org/10.3844/jmssp.2005.225.233.

[12] H.D. Kuhi, T. Porter, S. López, E. Kebreab, A.B. Strathe, A. Dumas, J. Dijkstra and J. France. A review of mathematical functions for the analysis of growth in poultry. World's Poultry Sci. J., 66:227-239, 2010. http://dx.doi.org/10.1017/S0043933910000280.

[13] H. Liao and D.W. Knight. Analytic stage-discharge formulae for flow in straight trapezoidal open channels. Adv. Water Resour., 30:2283-2295, 2007. http://dx.doi.org/10.1016/j.advwatres.2007.05.002.

[14] A.S. Martinez, R.S. Gonzalez and C.A.S. Tercariol. Continuous qrowth models in terms of generalized logarithm and exponential functions. Phys. A, 387:56795687, 2008. http://dx.doi.org/10.1016/j.physa.2008.06.015.

[15] M. Marusic and Z. Bajzer. Generalized two-parameter equation of growth. J. Math. Anal. Appl., 179:446-462, 1993.

http://dx.doi.org/10.1006/jmaa.1993.1361.

[16] P.S. Meyer and J.H. Ausubel. Carrying capacity: a model with logistically varying limits. Technological Forecasting and Social Change, 61:209-214, 1999. http://dx.doi.org/10.1016/S0040-1625(99)00022-0.

[17] P.S. Meyer, J.W. Yung and J.H. Ausubel. A primer on logistic growth and substitution: the mathematics of the Loglet Lab Software. Technological Forecasting and Social Change, 61:247-271, 1999.

http://dx.doi.org/10.1016/S0040-1625(99)00021-9.

[18] M.M. Mischan, S.Z. Pinho and L.R. Carvalho. Determination of a point sufficiently close to the asymptote in nonlinear growth functions. Sci. Agric. (Piracicaba, Braz.), 68:109-114, 2011. http://dx.doi.org/10.1590/S0103-90162011000100016.

[19] P.H. Morgan, L.P. Mercer and N.W. Flodin. General model for nutritional response of higher organisms. Proc. Nat. Acad. Sci. USA, 72:4327-4331, 1975. http://dx.doi.org/10.1073/pnas.72.11.4327. 
[20] J.D. Murray. Mathematical Biology. Springel, Berlin, 1989.

[21] A. Petersen-Overleir. Modelling stage-discharge relationships affected by hysteresis using the Jones formula and nonlinear regression. Hydrological Sci. J., 51:365-388, 2006. http://dx.doi.org/10.1623/hysj.51.3.365.

[22] M.S. Philip. Measuring Trees and Forests, 2nd ed. CAB International, Wallingford, UK, 1994.

[23] D.A. Ratkowsky. Nonlinear Regression Modeling. Marcel Dekker, New York, 1983.

[24] D.A. Ratkowsky. Handbook of Nonlinear Regression Models. Marcel Dekker, New York, 1989.

[25] J. Schnute. A versatile growth model with statistically stable parameters. Canad. J. Fisheries and Aquatic Sci., 38:1128-1140, 1981. http://dx.doi.org/10.1139/f81-153.

[26] R. Scitovski and D. Jukic. A method for solving the parameter identification problem for ordinary differential equations of the second order. Appl. Math. Comput., 74:273-291, 1996. http://dx.doi.org/10.1016/0096-3003(95)00098-4.

[27] R. Scitovski and S. Kosanovic. Rate of change in economics research. Economics Anal., 19:65-73, 1985.

[28] G.A.F. Seber and C.J. Wild. Nonlinear Regression. John Wiley \& Sons, New York, 1989.

[29] J.J. Shepherd and L. Stojkov. The logistic population model with slowly varying carrying capacity. ANZIAM J., 47:492-506, 2007.

[30] J. Tobin. Estimation of relationships for limited dependent variables. Econometrica, 26:24-36, 1958. http://dx.doi.org/10.2307/1907382.

[31] A. Tsoularis and J. Wallace. Analysis of logistic growth models. Math. Biosci., 179:21-55, 2002. http://dx.doi.org/10.1016/S0025-5564(02)00096-2.

[32] J.A. Westphal, D.B. Thompson, G.T. Stevens Jr. and C.N. Strauser. StageDischarge relations on the middle Mississippi River. J. Water Resour. Plann. Manage., 125:48-53, 1999. http://dx.doi.org/10.1061/(ASCE)0733-9496(1999)125:1(48).

[33] L. Yuancai, C.P. Marques and F.W. Macedo. Comparaison of Schnute's and Bertalanffy-Richards' growth function. Forest Ecology and Management, 96:283288, 1997. http://dx.doi.org/10.1016/S0378-1127(96)03966-7.

[34] B. Zeide. Analysis of growth equations. Forest Sci., 39:594-616, 1993.

[35] L. Zhang. Cross-validation of non-linear growth functions for modelling tree height-diameter relationships. Ann. Bot., 79:251-257, 1997. http://dx.doi.org/10.1006/anbo.1996.0334. 